\title{
Remoción de Cromo (VI) en Solución Acuosa por la Biomasa Celular de Paecilomyces sp.
}

Ismael Acosta, Juan F. Cárdenas, Diana Alvarado-Hernández y María G. Moctezuma-Zárate Universidad Autónoma de San Luis Potosí, Facultad de Ciencias Químicas, Centro de Investigación y de Estudios de Posgrado, Av. Dr. Manuel Nava No. 6, Zona Universitaria, 78320 San Luis Potosí, S.L.P.-México (e-mail: iacosta@uaslp.mx)

\section{Resumen}

El objetivo de este trabajo fue determinar la remoción de Cromo (VI) en solución por la biomasa celular del hongo contaminante ambiental Paecilomyces sp. por el método colorimétrico de la difenilcarbazida. La mayor bioadsorción fue a $\mathrm{pH}=1.0 \pm 0.2$, a $50^{\circ} \mathrm{C}$ durante 16 horas con $7.2 \mathrm{mg} / 200$ $\mathrm{mL}$ de concentración inicial de Cromo (VI) y $80 \mathrm{mg} / 200 \mathrm{~mL}$ de biomasa celular. Se concluye que la biomasa fúngica remueve adecuadamente Cromo $(\mathrm{VI})$ en solución y puede utilizarse para descontaminar nichos acuáticos contaminados, ya que $1 \mathrm{~g}$ de biomasa fúngica remueve $100 \mathrm{mg} / 200$ $\mathrm{mL}$ del metal a las tres horas de incubación.

Palabras clave: bioadsorción de cromo, biomasa fúngica, descontaminación de aguas, colorimetría

\section{Removal of Chromium (VI) from Aqueous Solutions by Fungal Biomass of Paecilomyces sp.}

\begin{abstract}
The objective of this work was to determine the removal of Chromium (VI) on the Paecilomyces sp.; fungal biomass, spectrophotometrically using diphenylcarbazide as the complexing agent. The highest adsorption was obtained at $\mathrm{pH}=1.0 \pm 0.2$, at $50^{\circ} \mathrm{C}$ after 16 hours of incubation, with 7.2 $\mathrm{mg} / 200 \mathrm{~mL}$ of initial concentration of Chromium (VI) and $80 \mathrm{mg} / 200 \mathrm{~mL}$ of cellular biomass. It was concluded that application of this biomass on the removal of $\mathrm{Cr}(\mathrm{VI})$ in aqueous solutions can be used since $1 \mathrm{~g}$ of fungal biomass remove $100 \mathrm{mg} / 200 \mathrm{~mL}$ of this metal after three hours of incubation.
\end{abstract}

Keywords: biosorption of chromium, fungal biomass, water decontamination, colorimetry 


\section{INTRODUCCIÓN}

Debido a las actividades industriales, como la producción de acero, minería, cemento y curtido de pieles, ciertas zonas de México, tienen altos niveles de cromo en suelo y agua (Armienta-Hernández y Rodríguez, 1995). El uso de lodos de aguas negras o de fertilizantes con diferentes concentraciones del catión, en algunas prácticas agronómicas, son otros de los factores contribuyentes a la contaminación ambiental por el metal (Acevedo-Aguilar et al., 2006). El cromo se encuentra presente en agua y suelo principalmente en dos formas de oxidación: $\mathrm{Cr}$ (III) o $\mathrm{Cr}(\mathrm{VI})$, aunque también puede encontrarse como óxido de cromo, sulfato de cromo, trióxido de cromo, ácido crómico y dicromato (Zouboulis et al., 1995). En presencia de la materia orgánica, el $\mathrm{Cr}$ (VI) presente en aguas y suelos es reducido a $\mathrm{Cr}$ (III); sin embargo, las altas concentraciones del ión en estado hexavalente pueden sobrepasar esta capacidad de reducción, lo que impediría su adecuada eliminación (Cervantes et al., 2001). Pese a que el cromo es un elemento esencial para hombres y animales, niveles elevados de este metal (15 $\mathrm{gg}$ en agua de ríos y $0.10 \mathrm{mg} / \mathrm{L}$ en agua potable) resultan tóxicos en estos seres vivos. Particularmente, el $\mathrm{Cr}(\mathrm{VI})$ tiene efectos carcinogénicos en animales y mutagénicos en humanos y bacterias (Cervantes et al., 2001). En las aguas residuales, el $\mathrm{Cr}(\mathrm{VI})$, se encuentra en solución como $\mathrm{CrO}_{4}{ }^{2-}$ (Cotton y Wilkinson, 1980), el cual puede removérsele por reducción, por precipitación química, por adsorción y por intercambio iónico (Cervantes et al., 2001). El proceso más utilizado es la adición de un agente reductor que convierta el $\mathrm{Cr}$ (VI) a $\mathrm{Cr}$ (III) y posteriormente se le precipita con soluciones básicas a $\mathrm{Cr}(\mathrm{OH})_{3}$ (Campos et al., 1995). Recientemente, se ha estudiado el uso de metodologías alternativas, como la reducción de $\mathrm{Cr}(\mathrm{VI})$ a $\mathrm{Cr}$ (III) por los agentes Pseudomonas sp. (Maclean y Beveridge, 2001), Pseudomonas putida (Park et al., 2000), Acidithiobacillus ferrooxidans (Allegretti et al., 2006), Candida maltosa (Ramírez-Ramírez et al., 2004), Penicillium sp. y Aspergillus sp. (Acevedo-Aguilar et al., 2006), qué son hongos, bacterias y levaduras y la bioadsorción del mismo por biomasas fúngicas como: Cryptococcus neoformans y Helmintosporium sp. (Acosta et al., 2004), Aspergillus niger (Kapoor et al., 1999; Park et al., 2005), Rhizopus arrhizus (Sag y Aktay, 2002) y Mucor hiemalis (Tewari et al., 2005).

El objetivo de este trabajo es estudiar la remoción de Cromo (VI) en solución por la biomasa fúngica del hongo contaminante ambiental Paecilomyces sp. para la descontaminación de aguas residuales que contengan dicho metal.

\section{METODOLOGÍA}

\section{Biomasa fúngica}

Se utilizó la biomasa celular del hongo contaminante ambiental Paecilomyces sp.; resistente a $\mathrm{Cr}$ (VI), aislado del aire de una zona cercana a la Facultad de Ciencias Químicas de la UASLP, San Luís Potosí, S.L.P, México (temperatura media anual de $18.6^{\circ} \mathrm{C}$ y 1860 metros sobre el nivel del mar), a partir de medios de Agar Extracto de Malta adicionados con 500 ppm de $\mathrm{Cr}(\mathrm{VI})$. El crecimiento del hongo se llevó a cabo inoculando $1 \times 10^{6}$ células $/ \mathrm{mL}$ en caldo tioglicolato $(8 \mathrm{~g} / \mathrm{L})$, a $28^{\circ} \mathrm{C}$ con agitación constante (100 rpm). 4 días después de la incubación, se obtuvo la biomasa por filtración en papel Whatman No. 2. Posteriormente se centrífugo ( $3000 \mathrm{rpm}, 5 \mathrm{~min}$ ), se lavó 3 veces con agua tridesionizada, se secó $\left(80^{\circ} \mathrm{C}, 12 \mathrm{~h}\right)$ en estufa bacteriológica, se molió en mortero y se guardó en frascos de vidrio ámbar a temperatura ambiente hasta su utilización.

\section{Soluciones de $\mathrm{Cr}(\mathrm{VI})$}

Se trabajó con $200 \mathrm{~mL}$ de una solución de $7.2 \mathrm{mg} / \mathrm{L}$ de concentración de $\mathrm{Cr}(\mathrm{VI})$ obtenida por dilución de una solución patrón de $71.86 \mathrm{mg} / \mathrm{L}$ preparada en agua tridesionizada a partir de $\mathrm{K}_{2} \mathrm{CrO}_{4}$. Se ajusto el pH de la dilución a analizar con $\mathrm{H}_{2} \mathrm{SO}_{4} 1 \mathrm{M}$ y/o NaOH $1 \mathrm{M}$, antes de adicionarla a la biomasa celular.

\section{Estudios de remoción}

$80 \mathrm{mg}$ de biomasa celular se mezclaron con $200 \mathrm{~mL}$ de una solución de $7.2 \mathrm{mg} / \mathrm{L}$ de concentración del metal $\left(\mathrm{pH}=2.0+/-0.2\right.$ y $\left.28^{\circ} \mathrm{C}\right)$ y se incubaron con agitación constante $(100 \mathrm{rpm})$ durante 24 a 48 
$\mathrm{h}$, tomando cada $2 \mathrm{~h}$ y en condiciones estériles, alícuotas de $5 \mathrm{~mL}$ cada una, las cuales se centrifugaron a $3000 \mathrm{rpm}$ (5 $\mathrm{min})$, y al sobrenadante respectivo se le determinó la concentración de $\mathrm{Cr}(\mathrm{VI})$, utilizando el método colorimétrico de la difenilcarbazida (desarrollo de coloración rosa violeta). A las muestras $(5 \mathrm{~mL})$, que contenían de 0 a $7.2 \mathrm{mg} / \mathrm{L}$ de $\mathrm{Cr}(\mathrm{VI})$ se les añadió $0.5 \mathrm{~mL}$ de una solución de $\mathrm{H}_{2} \mathrm{SO}_{4} 1: 1(\mathrm{v} / \mathrm{v}), 0.1 \mathrm{~mL}$ de ácido fosfórico al $85 \%(\mathrm{v} / \mathrm{v})$ y $1.0 \mathrm{~mL}$ de difenilcarbazida en etanol absoluto, se incubaron durante 10 minutos a temperatura ambiente, y se les leyó la absorbancia a una longitud de onda de $540 \mathrm{~nm}$ (Greenberg et al., 1992). Todos los experimentos se realizaron 3 veces y por triplicado.

\section{RESULTADOS Y DISCUSIÓN}

La literatura (Acosta et. al., 2004) reporta un tiempo de incubación de 24 h cuando se trabajó con Cryptococcus neoformans y Helmintosporium sp.; a pH de 2.0 y 5 días para Aspergillus. niger (Park et al., 2005), este último con $10 \mathrm{~g} / \mathrm{L}$ de biomasa, y al mismo $\mathrm{pH}$ de 2.0. Cambios en la permeabilidad de la pared celular fúngica, de origen desconocido, podrían explicar en parte las diferencias encontradas en el tiempo de incubación, proporcionando mayor o menor exposición de los grupos funcionales de la pared celular de la biomasa analizada (Kratochvil y Volesky, 1998).

La figura 1 muestra la influencia del $\mathrm{pH}$ inicial sobre la eficiencia de remoción. La mayor actividad se evidenció a $\mathrm{pH} 1.0+/-0.2$, pues a las $52 \mathrm{~h}$ se remueve el $100 \%$ del metal. Se ha reportado un $\mathrm{pH}$ óptimo para la remoción de $\mathrm{Cr}(\mathrm{VI})$ por los hongos Rhizopus arrhizus y Saccharomyces cerevisiae en un rango de 1.5-2.5, a las $4 \mathrm{~h}$ (Nourbakhsh et al., 1994), aunque la mayoría indican un pH óptimo de remoción en un rango de 2.0 a 3.0 (Tewari et al., 2005; con Mucor hiemalis; Sag y Aktay, 2002; para Rhizopus arrhizus, ambos a las $24 \mathrm{~h}$; Bai y Abraham, 2001; con Rhizopus nigricans, a las $8 \mathrm{~h}$ ). El $\mathrm{Cr}$ (VI) se encuentra como $\mathrm{HCrO}_{4}{ }^{-} \mathrm{Cr}_{2} \mathrm{O7}^{2-}, \mathrm{CrO}_{4} 2-, \mathrm{Cr}_{4} \mathrm{O}_{13}{ }^{2-}, \mathrm{Cr}_{3} \mathrm{O}_{10}{ }^{2-}$ (Rollinson, 1973). Una baja en el $\mathrm{pH}$ causa la protonación de la superficie del adsorbente, lo que induce una fuerte atracción por los iones $\mathrm{Cr}(\mathrm{VI})$ de la solución cargados negativamente, por lo que la bioadsorción aumenta al aumentar la acidez de la solución. Pero, cuando el pH aumenta, se incrementa la concentración de iones $\mathrm{OH}^{-}$, induciendo cambios en la superficie del adsorbente, impidiendo la bioadsorción de los iones $\mathrm{Cr}(\mathrm{VI})$ cargados negativamente, lo cual disminuye la adsorción del metal a valores de $\mathrm{pH}$ altos. AcevedoAguilar et al. (2006) reportaron que si bien el Cr (VI) se consigue eliminar por cepas autóctonas de hongos filamentosos, la mayor parte del catión puede ser reducido a $\mathrm{Cr}$ (III).

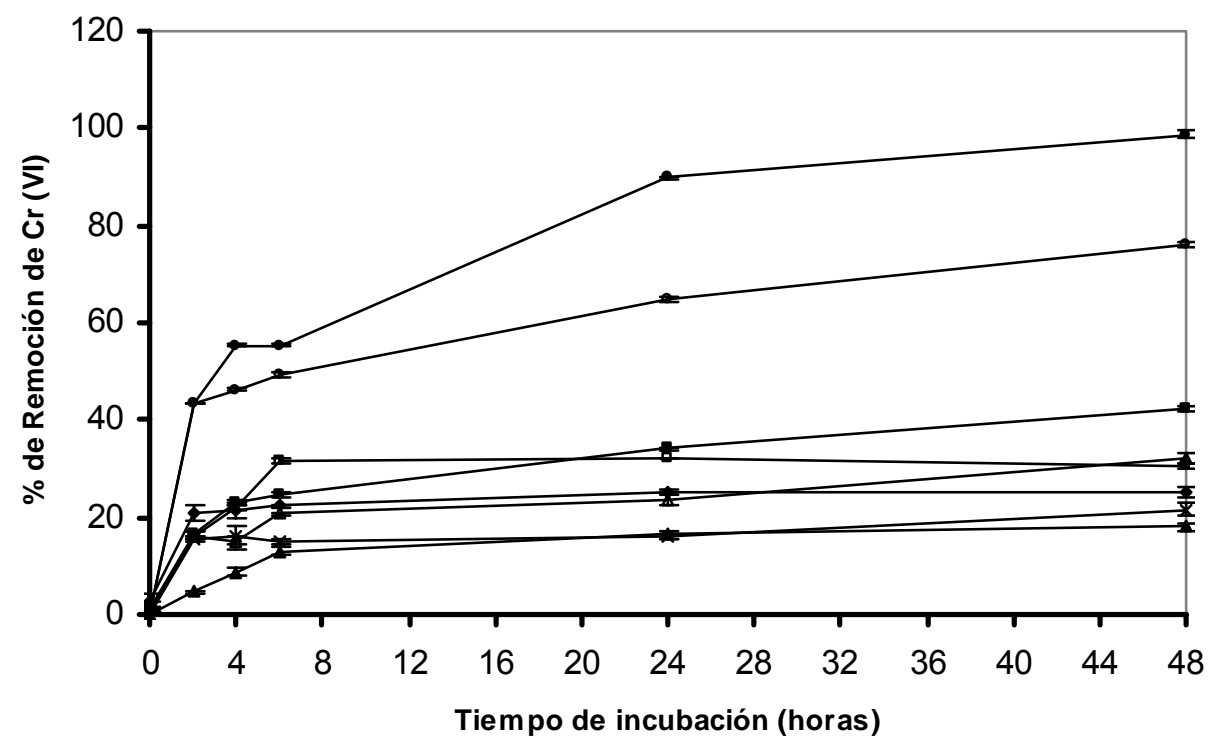

Fig. 1: Efecto del pH sobre la bioadsorción de Cromo (VI). 7.2 mg/100 $\mathrm{mL}$ con $80 \mathrm{mg}$ de biomasa, $28^{\circ} \mathrm{C}$, con agitación constante (100 rpm). \% de remoción de $\mathrm{Cr}(\mathrm{VI})$; $\mathrm{pH} 1.0 \bullet$, pH 2.0 ○, pH 3.0 घ, pH 4.0 口, pH 5.0 $\times, \mathrm{pH} 6.0 \Delta, \mathrm{pH} 7.0 \Delta, \mathrm{pH} 8.0$ •. (Datos tomados con una $\mathrm{n}=3$ y una media +/- error estándar). 


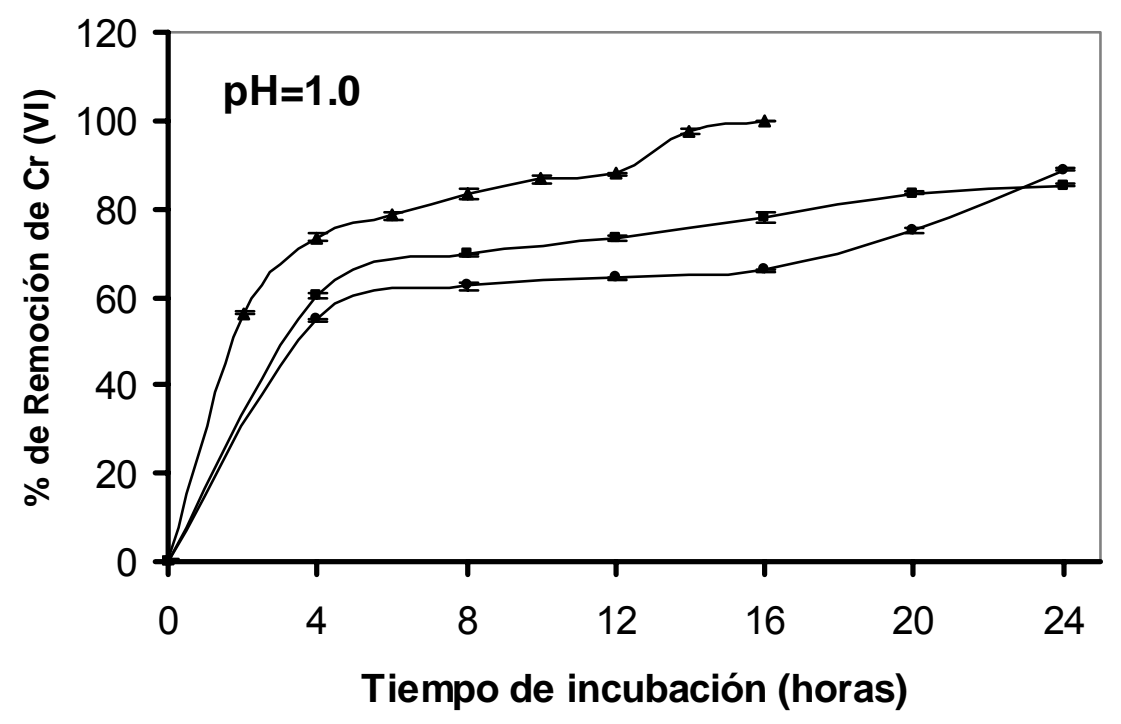

Fig. 2: Efecto de la temperatura de incubación sobre la bioadsorción de Cromo (VI). $7.2 \mathrm{mg} / 100 \mathrm{~mL}$ con $80 \mathrm{mg}$ de biomasa, con agitación constante $(100 \mathrm{rpm}) . \%$ de remoción de $\mathrm{Cr}(\mathrm{VI}): 28^{\circ} \mathrm{C} \bullet$, $37^{\circ} \mathrm{Cm}, 50^{\circ} \mathrm{C} \Delta$ (Datos tomados con una $\mathrm{n}=3$ y una media $+/-$ error estándar).

En lo que tiene que ver con la temperatura, la más alta remoción se observó a $50^{\circ} \mathrm{C}$ (Figura 2), pues a las $16 \mathrm{~h}$ se remueve el $100 \%$ de $\mathrm{Cr}(\mathrm{VI})$ en solución; resultados que son coincidentes con los de Park et al. (2005), quienes observaron que a $45^{\circ} \mathrm{C}$ y $24 \mathrm{~h}$, ocurre la adsorción del mismo metal para Aspergillus niger, pero difieren de los $35^{\circ} \mathrm{C}$ y $24 \mathrm{~h}$ reportados por Sag y Aktay (2002) para Rhizopus arrhizus. El incremento en la temperatura, aumenta la velocidad de remoción de $\mathrm{Cr}$ (VI) y disminuye el tiempo de contacto requerido para la completa remoción del metal, por incrementar la velocidad de reacción redox (Wittbrodt y Palmer, 1996).

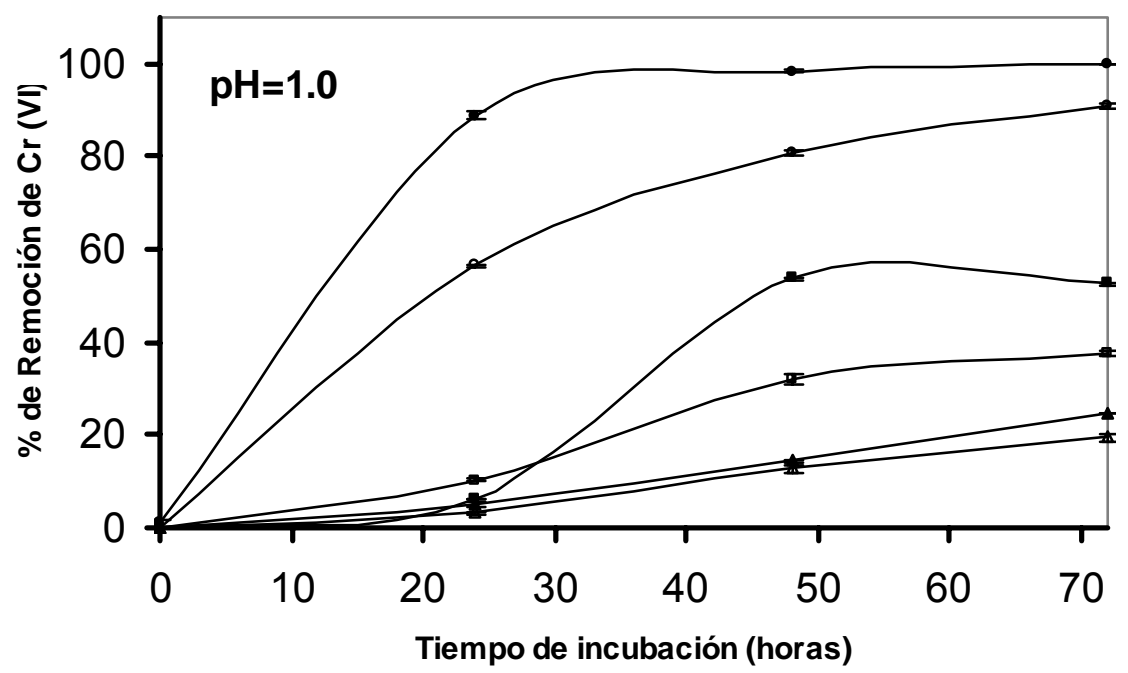

Fig. 3: Efecto de la concentración inicial del metal sobre la bioadsorción de Cromo (VI) en solución. $80 \mathrm{mg}$ de biomasa/ $100 \mathrm{~mL}$, $50^{\circ} \mathrm{C}$, con agitación constante (100 rpm). Concentración de $\mathrm{Cr}(\mathrm{VI})$ : $10 \mathrm{mg} / \mathrm{L} \bullet, 20 \mathrm{mg} / \mathrm{L} \circ, 30 \mathrm{mg} / \mathrm{L}$ !, $40 \mathrm{mg} / \mathrm{L} \square, 50 \mathrm{mg} / \mathrm{L} \boldsymbol{\Delta}, 60 \mathrm{mg} / \mathrm{L} \Delta$ (Datos tomados con una $n=3$ y una media $+/$ - error estándar).

A bajas concentraciones del metal $(10$ y $20 \mathrm{mg} / \mathrm{L})$, la biomasa estudiada, mostró las mejores respuestas de remoción, adsorbiendo el $100 \%$ a las 52 y $96 \mathrm{~h}$, respectivamente, mientras que a 60 $\mathrm{mg} / \mathrm{L}$ del metal sólo se remueve un $20 \%$ de Cr (VI) (Figura 3). Algunos autores (Tewari et. al., 2005; Bai y Abraham, 2001), sostienen que la cantidad de metal eliminado por las biomasas de diferentes 


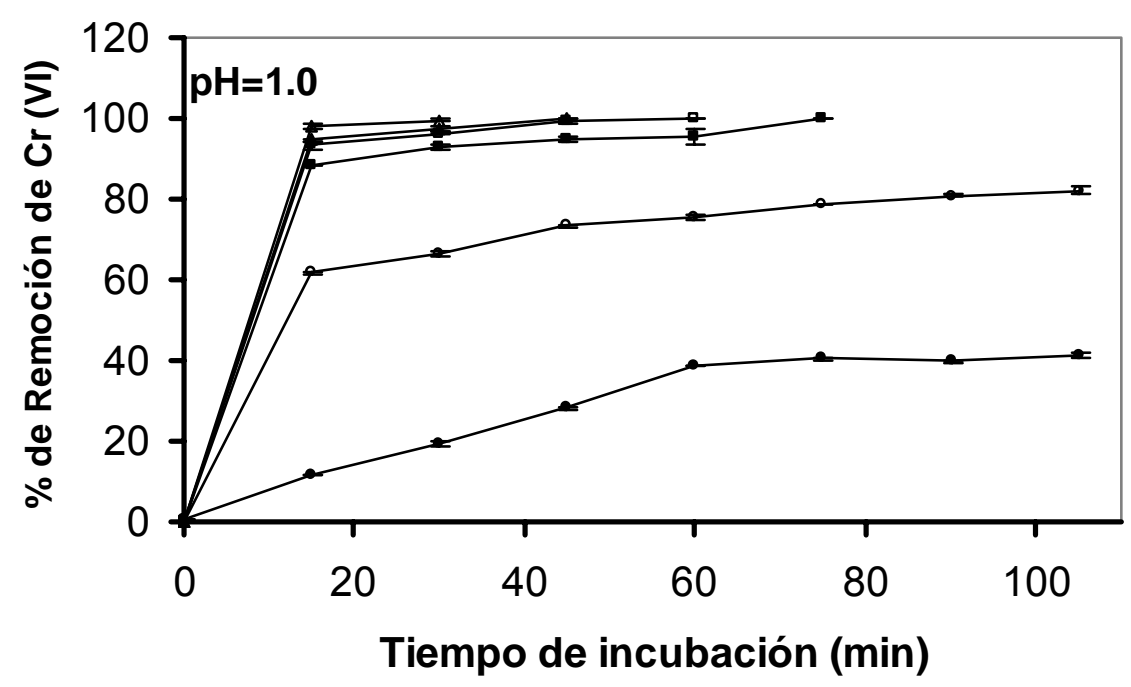

Fig. 4: Efecto de la concentración inicial de biomasa celular sobre la bioadsorción de Cromo ( $\mathrm{VI}$ ) en solución. $7.2 \mathrm{mg} / 100 \mathrm{~mL}, 50^{\circ} \mathrm{C}$, con agitación constante (100 rpm). Biomasa: $100 \mathrm{mg} \bullet, 200 \mathrm{mg}$ ○, $400 \mathrm{mg}$ -, $600 \mathrm{mg} \square, 800 \mathrm{mg} \boldsymbol{\Delta}, 1000 \mathrm{mg} \Delta$ (Datos tomados con una $\mathrm{n}=3 \mathrm{y}$ una media +/- error estándar).

microorganismos, tales como Mucor hiemalis y Rhizopus nigricans aumenta en proporción directa con el incremento de la concentración del ión metálico en solución. De nuevo se encuentran discrepancias con los resultados de este trabajo, debido a que la biomasa utilizada en el estudio mostró la mayor capacidad de remoción a bajas concentraciones (10 y $20 \mathrm{mg} / \mathrm{L}$ ). En la figura 4, se muestra que al aumentar la cantidad de biomasa, también se incrementa la remoción de $\mathrm{Cr}(\mathrm{VI})$ en solución, pues hay más sitios de bioadsorción del metal. Resultados similares se han reportado para Mucor hiemalis y Rhizopus nigricans, aunque con $10 \mathrm{~g}$ de biomasa (Tewari et. al., 2005; Bai y Abraham, 2001). Cabe destacar que la metodología efectuada es muy reproducible en cada uno de los ensayos realizados y lo podemos observar con la determinación del error estándar en cada una de las graficas.

\section{CONCLUSIONES}

La biomasa fúngica analizada remueve eficientemente $\mathrm{Cr}(\mathrm{VI})$ en solución, y el hongo puede utilizarse para eliminar el $\mathrm{Cr}(\mathrm{VI})$ presente en aguas residuales industriales, pues presenta mayor capacidad a otras biomasas reportadas en la literatura. La aplicación de la remoción de metales pesados en solución, para la purificación de aguas residuales presenta un gran potencial, pues las biomasas fúngicas son naturales, se pueden obtener en grandes cantidades, son económicas, y pueden remover selectivamente diferentes iones metálicos de soluciones acuosas (Kratochvil y Volesky, 1998).

\section{AGRADECIMIENTOS}

Se agradece a la UASLP por su contribución con este trabajo mediante el Convenio C06-FAI-03-7.10 de la UASLP

\section{REFERENCIAS}

Acevedo-Aguilar, F., A. y otros 9 autores; Hexavalent chromium removal in Vitro and from industrial wastes, using chromate-resistant strains of filamentous fungi indigenous to contaminated wastes, Canadian Journal of Microbiology: 52(9): 809-815 (2006).

Acosta, I.,X. Rodríguez, C. Gutiérrez y M.G. Moctezuma; Biosorption of Chromium (VI) from aqueous solutions onto fungal biomass, Bioinorganic Chemistry Applications: 2 (1,2) 1-7 (2004). 
Allegretti. P., J. Furlong y E. Donati; The role of higher polythionates in the reduction of chromium (VI) by Acidithiobacillus and Thiobacillus cultures, Journal of Biotechnology: 122(1) 55-61 (2006).

Armienta-Hernández, M. y R. Rodríguez Castillo; Environmental exposure to Chromium compounds in the valley of León, México, Environmental Health Perspectives: 103, 47- 51(1995).

Bai, R.S. y T.E. Abraham; Biosorption of $\mathrm{Cr}(\mathrm{VI})$ from aqueous solution by Rhizopus nigricans, Bioresource Technology: 79, 73-81 (2001).

Campos, J., M. Martínez-Pacheco y C. Cervantes, Hexavalent chromium reduction by a chromateresistant Bacillus sp strain, Antonie van Leeuwenhoek: 68, 203-208 (1995).

Cervantes, C., J. y otros 6 autores; Interactions of chromium with microorganisms and plants, FEMS Microbiology Review 25, 333-347 (2001).

Cotton, F.A. y G. Wilkinson; Advanced Inorganic Chemistry, $4^{\mathrm{a}}$ Ed. Chichester, Uk; John Wiley\&Sons, 376-379 (1980).

Greenberg, A.E., L.S. Clesceri y A.D. Eaton; Standard methods for the examination of water and wastewater, $18^{\mathrm{a}}$ ed. American Public Health Association, Washington, D.C. 3.58-3.60 (1992).

Kapoor, A., T. Viraraghavan, y D.R. Cullimore; Removal of heavy metals using the fungus Aspergillus niger, Bioresource Technology: 70, 95-104 (1999).

Kratochvil, D. y B. Volesky; Advances in the biosorption of heavy metals, TIBTECH: 16, 291- 300 (1998).

McLean, J. y T.J. Beveridge; Chromate reduction by a pseudomonad isolated from a site contaminated with chromated copper arsenate, Appl. Environ. Microbiology: 67, 1076-1084 (2001).

Park, C.H., y otros 4 autores; Purification to homogeneity and characterization of a novel Pseudomona putida chromate reductase, Appl. Environ. Microbiology: 66(5), 1788-1795 (2000).

Park, D., Y. Yun, J. Jo y J.M., Park; Mechanism of hexavalent chromium removal by dead fungal biomass of Aspergillus niger. Water Research: 39, 533-540 (2005).

Ramírez-Ramírez, R. y otros siete autores; $\mathrm{Cr}(\mathrm{VI})$ reduction in a Chromate-resistant strain of Candida maltose isolated from the leather industry, Antonie van Leeuwenhoek: 85, 63-68 (2004).

Rollinson, C.L.; Chromium, molybdenum and tungsten, in: T. Dickson (ed), Comprehensive Organic Chemistry, $3^{\mathrm{a}}$. ed. Pergamon Press, Oxford, UK, 691-694 (1973).

Sag, Y. e Y. Aktay; Kinetic studies on sorption of $\mathrm{Cr}(\mathrm{VI})$ and $\mathrm{CU}(\mathrm{II})$ ions by chitin, chitosan and Rhizopus arrhizus. Biochemical Engineering Journal: 12, 143-153 (2002).

Tewari, N., P. Vasudevan, y B. Guha; Study on biosorption of $\operatorname{Cr}($ VI) by Mucor hiemalis, Biochemical Engineering Journal: 23, 185-192 (2005).

Wittbrodt, P.R. y C.D. Palmer; Effect of temperature, ion strength, background electrolytes, and Fe(III) on the reduction of hexavalent chromium by soil humic substances, Environmental Science Technology: 30 (8), 2470-2477 (1996).

Zouboulis, A.I., K.A. Kydros y K.A. Matis; Removal of hexavalent Chromium anions from solutions by pyrite fines, Water Research: 29 (7), 1755-1760 (1995). 